\title{
The multidisciplinary team plays an important role in the prediction of small solitary pulmonary nodules: a propensity-score-matching study
}

\author{
Chaoyuan Liu, Lishu Zhao, Fang Wu, Yeqian Feng, Rong Jiang, Chunhong Hu \\ Department of Oncology, The Second Xiangya Hospital, Central South University, Changsha 410011, China \\ Contributions: (I) Conception and design: C Liu, C Hu; (II) Administrative support: C Hu; (III) Provision of study materials or patients: F Wu, R \\ Jiang; (IV) Collection and assembly of data: L Zhao; (V) Data analysis and interpretation: C Liu, Y Feng; (VI) Manuscript writing: All authors; (VII) \\ Final approval of manuscript: All authors. \\ Correspondence to: Chunhong Hu. Department of Oncology, The Second Xiangya Hospital, Central South University, No. 139 Middle Renmin Road, \\ Changsha 410011, China. Email: huchunhong@csu.edu.cn.
}

Background: According to guidelines, it is recommended that pulmonary nodules be discussed by a multidisciplinary team (MDT); however, the evidence for the effectiveness of MDT is sparse. To demonstrate the importance of the involvement of an MDT for the prediction of small solitary pulmonary nodules, we conducted this retrospective study.

Methods: The patient database of those who attended our MDT and the electronic medical record system of our hospital was used; we collected all the data from patients found with small solitary pulmonary nodules $(\leq 2 \mathrm{~cm})$, which were suspected as malignant and who received a resection of the nodules. We summarized their characteristics and analyzed them, and then compared the post-operation pathological diagnosis of the patients who attended an MDT to those who did not participate in an MDT during the same period (20172019.2). We also collected the follow-up data. Propensity-score-matching was utilized during the process of analysis to get a more reliable conclusion.

Results: Most of the qualified patients were female. Most of the small solitary pulmonary nodules $(\leq 2 \mathrm{~cm}$ ) were adenocarcinoma and located on the right upper lobe. There were no differences in the SUV value between malignant nodules and benign nodules. After propensity-score matching, the total positive prediction value of small solitary pulmonary nodules $(\leq 2 \mathrm{~cm})$ without an MDT was $69.4 \%$, while that with MDT was $77.6 \%$; the difference was not significant with a $\mathrm{P}$ value of 0.30 . The negative predictive value of MDT was $76.2 \%$.

Conclusions: In developing countries, small solitary pulmonary nodules tend to be more correctly diagnosed with MDT.

Keywords: Small solitary pulmonary nodule; multidisciplinary team (MDT); propensity-score-matching; positive prediction value; negative prediction value

Submitted Aug 29, 2019. Accepted for publication Nov 15, 2019.

doi: 10.21037/atm.2019.11.125

View this article at: http://dx.doi.org/10.21037/atm.2019.11.125

\section{Introduction}

Lung cancer is still the leading cause of cancer-related deaths worldwide $(1,2)$. The 5 -year survival rate for lung cancer patients at any stage is $18 \%$, and the survival rate increases as the stage declines. For example, stage IV has a
$2 \% 5$-year survival rate, while stage IA boasts a $73 \% 5$-year survival rate after complete resection (3). Adenocarcinoma in situ, the 5-year disease-free survival after resection can even reach $100 \%$ (4). The earlier we detect and treat lung cancer, the better the prognosis. CT screening is a 
frequently used strategy for detecting early stage lung cancer. In the US National Lung Screening Trial, researchers found that CT screening could reduce lung cancer mortality by up to $20 \%$ (5).

A pulmonary nodule is defined as a single, wellcircumscribed, spherical with a diameter $\leq 3 \mathrm{~cm}$, surrounded by lung tissue, and which is not associated with hilar enlargement, atelectasis, or pleural effusion (6). With the popularization of computed tomography (CT) screening worldwide, more and more pulmonary nodules have been found (7). Most of them are benign, but the primary concern is in the identification of malignancy. The right prediction could avoid unnecessary surgery in patients with benign lesions (8). All modalities show lower sensitivity for nodules, whose diameter is $\leq 2 \mathrm{~cm}$ and whose sensitivity is about $33 \%$ (9). How should we tackle these nodules? How should we avoid over-diagnosis and overtreatment? A series of international guidelines were produced to address this universal problem $(10,11)$. In the guidelines, which are mostly published in developed countries, pulmonary nodules are highly recommended to be discussed by a multidisciplinary team (MDT). However, the recommendation is built on intuition rather than solid evidence. In some developing countries, such as China, where pulmonary tuberculosis is much more prevalent than western developed countries (12), is an MDT still necessary for patients with small pulmonary nodules? Can the patients receive a correct diagnosis for their nodules using an MDT? To answer these questions and to measure the benefits of MDT for pulmonary nodules in the real world, we conducted a retrospective study in our department, a top 20 cancer institute in China.

\section{Methods}

\section{Introduction of our center and the MDT}

Our center and the MDT were introduced in our earlier study (13). Both our hospital and our research center currently ranks in the top 20 of comprehensive tertiary care hospitals and cancer institutes in China.

The MDT for thoracic tumors in our center was established in December 2016. It consists of a full-time secretary who is an oncology physician and a senior oncologist who takes charge of the MDT. An MDT meeting is conducted once a week. The director of our center serves as a chairman of the MDT meeting and provides a strategic conclusion for the team. The patients whose cases are presented at our MDT can be referred by any specialists from any department of our hospital. The patients with lung nodules who are recommended to MDT should meet the following criteria: (I) having a nodule for which getting a biopsy before operation is relatively difficult; (II) different opinions from two specialists about the diagnosis or different treatment recommendations for the same patient. Customarily, once the patient arrives at the office of our MDT, the secretary will record their case history, perform the necessary examinations, and collect any relevant tests or radiological reports. The senior oncologist in charge will analyze these materials and decide which specialists from which departments are needed to attend the upcoming MDT meeting. Usually, medical oncologists, radiation oncologists, pulmonologists, thoracic surgeons, radiologists, pathologists, and nurses are invited. Before the meeting, all the collected medical information will be announced in the MDT We-Chat group. At the meeting, the secretary and the senior oncologist present these cases sequentially, and all the specialists discuss together and come to the most appropriate diagnosis and treatment recommendations for each patient. The patients wait outside the meeting room and will be invited into the room and receive some queries and examinations during the meeting. After the meeting, the secretary and the senior oncologist in charge will explain the diagnosis and treatment recommended by the MDT to the patients or their caregivers in detail. The nurse will make the necessary consoling and procedure instructions.

\section{Study population}

The study protocol was approved by the medical ethics committee of the Second Xiangya Hospital. There were two groups formed from a Chinese population. The first group (MDT group) was identified from the medical database of patients who attended our MDT and the second group (non-MDT group) was identified from the electronic medical record system of the whole hospital. The common inclusion criteria were as follows: (I) an admission time between January 1, 2017, and February 28, 2019; (II) a diagnosis made by MDT in the MDT group and by the doctor in charge of the non-MDT group included "pulmonary nodule" with suspected malignancy and recommended surgery; (III) a patient receiving an operation for the lung nodule. The exclusion criteria were as follows: (I) a history of any cancer; (II) a patient receiving a pathological diagnosis before surgery; (III) a patient who had attended our MDT would also be excluded from the 


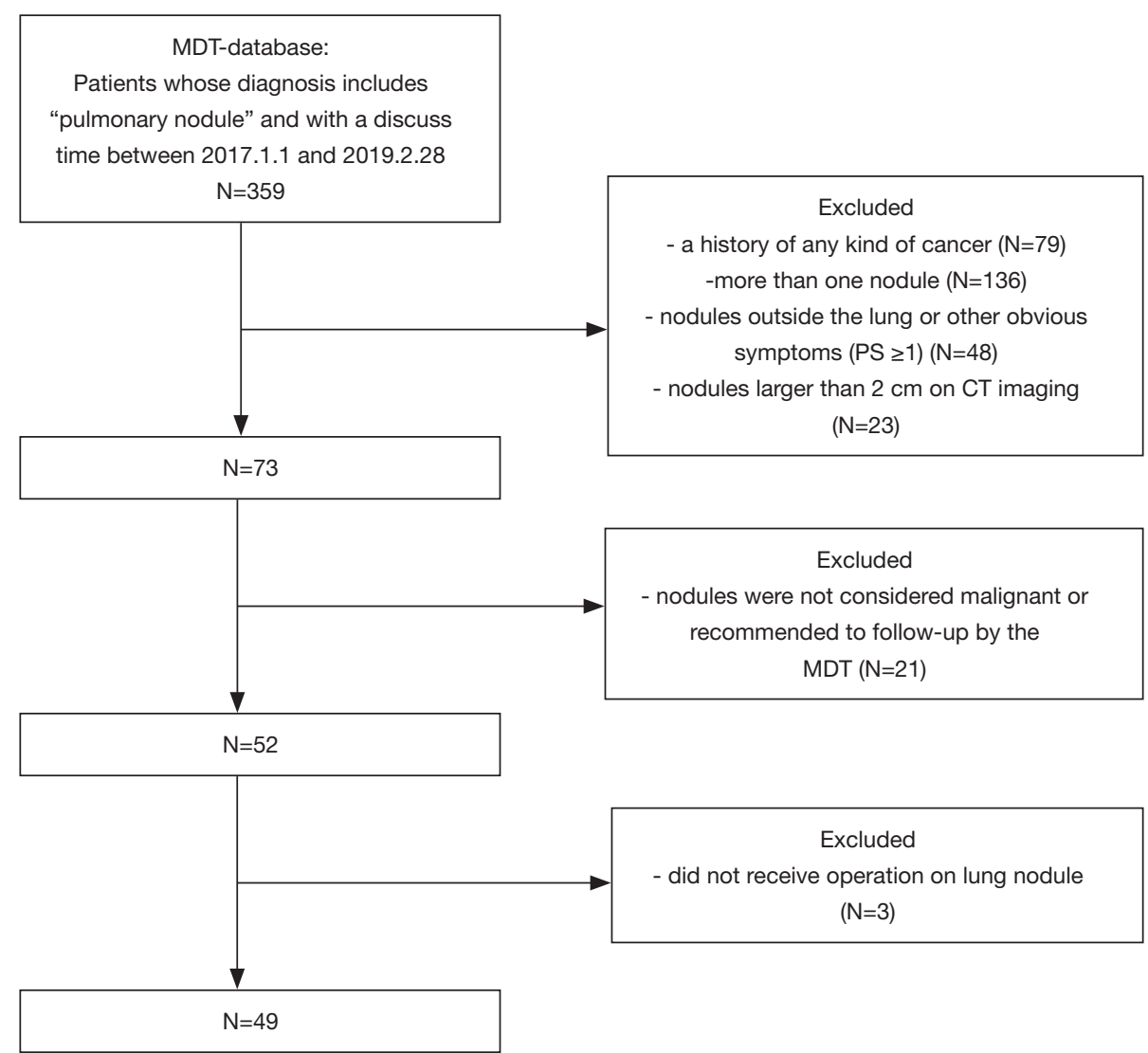

Figure 1 The flowchart of patient selection of MDT group. MDT, multidisciplinary team.

non-MDT group. Figures 1 and 2 show the flow of our selection for the two groups of the study population.

\section{Statistical methods}

To compare the difference between the MDT group and the non-MDT group, independent-sample tests were used for continuous variables that were normally distributed, and the results were expressed as the average \pm SD. For continuous variables that were not normally distributed, we used independent-sample and non-parametric tests, and the results were expressed with a median with corresponding interquartile range $(\mathrm{Q} 1, \mathrm{Q} 3)$. For categorical variables, we used the $\chi^{2}$ test, and values were presented as proportions in percentages. All statistical analyses were 2 -sided, and $\mathrm{P}<0.05$ was considered statistically significant. The analysis was conducted with a software called IBM SPSS Statistics 23.

EmpowerStats ( Solutions, Inc., Boston, MA, R 3.4.3) was adopted to perform propensity score matching based on the following confounding factors: gender, age, nodule type, nodule size, nodule position, spicule sign, and smoking history.

\section{Results}

\section{Characteristics of the study population and pulmonary nodules}

All the enrolled participants underwent an operation and successfully received a pathological diagnosis, none died during the operation. The basic demographic characteristic and the characteristics of the pulmonary nodules of two groups of the population before matching are shown in Table 1 (grouped by MDT and non-MDT) and Table 2 (grouped by malignant and benign pathology). There were 360 patients with 360 nodules included, with most of them being female (55.6\%), without a family malignancy history $(91.9 \%)$ or a history of smoking $(78.9 \%)$. Most of them did not undergo a PET-CT scan (77.2\%), and 


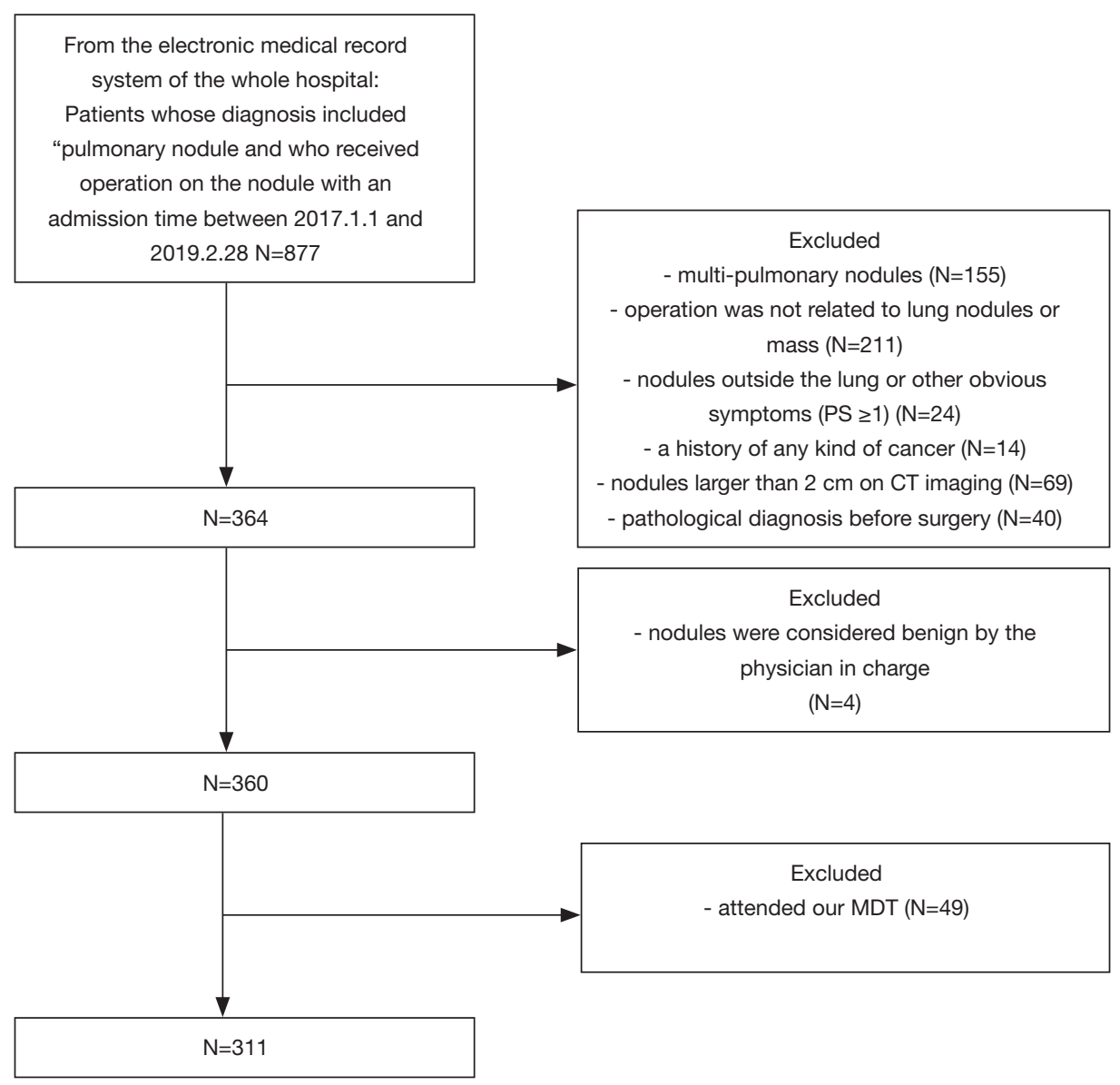

Figure 2 The flowchart of patient selection of non-MDT group. MDT, multidisciplinary team.

most nodules were in the right upper lobe of the lung (37.5\%). In the nodules which had PET-CT, most of the SUV values were below 2.5 in both the malignant group and benign group (98.2\% and $92.0 \%$ respectively). Well-/ moderately differentiated adenocarcinoma was the major pathology type (41.4\%). We propensity-score matched 98 patients from the non-MDT group with 49 patients from the MDT group (2:1). The demographic characteristic and the characteristics of the lung nodules after matching are summarized in Table 3 (grouped by MDT and non-MDT) and Table 4 (grouped by malignant and benign pathology) The characteristics of the lung nodules grouped by SUV value are shown in Table 5 .

\section{Comparison between MDT/non-MDT diagnosis and pathological diagnosis}

The positive predictive value (PPV) based on variables after propensity-score matching between the non-MDT and
MDT groups are shown in Table 6. We can see that most of the positive prediction value (PPV) based on variables in the MDT group are higher than those of the non-MDT group except in sub-solid nodules, right low lobe, with spicule, however, the p-values were all above 0.05 . In total, as shown in Tables 2 and 4, before matching, the positive prediction rate of MDT $(77.6 \%)$ was $7.2 \%$ higher than that in the non-MDT (70.4\%). After matching, the gap was enlarged to $8.2 \%$, with the positive prediction rate of the MDT being $77.6 \%$, with the non-MDT being $69.4 \%$; however, the $\mathrm{P}$ values were all above 0.05 .

\section{Results of follow-up in the MDT group}

The characteristics of the people who were recommended to follow-up in the MDT group are shown in Table 7. In the table, we can see that the follow-up time is 12.0 months (10.0-19.0 months), 2 patients were lost to follow-up, and the negative predictive value (NPV) in the MDT group was about $76.2 \%$. 
Table 1 Clinical characteristics of the 2 cohorts of patients with small solitary pulmonary nodules identified for propensity score matching (before matching)

\begin{tabular}{|c|c|c|c|c|}
\hline Characteristics & Total & Non-MDT & MDT & $P$ value \\
\hline Age (years), median (Q1-Q3) & $54.0(48.0-62.2)$ & $54.0(47.0-63.0)$ & $55.0(49.0-61.0)$ & 0.689 \\
\hline Nodule size (mm), median (Q1-Q3) & $13.0(9.5-16.0)$ & $13.0(10.0-17.0)$ & $11.0(9.0-15.0)$ & 0.015 \\
\hline Gender (n, \%) & & & & 0.139 \\
\hline Female & $200(55.6)$ & $168(54.0)$ & $32(65.3)$ & \\
\hline History of smoking (n, \%) & & & & 0.377 \\
\hline No & $284(78.9)$ & $243(78.1)$ & $41(83.7)$ & \\
\hline Yes & $76(21.1)$ & $68(21.9)$ & $8(16.3)$ & \\
\hline Yes & $29(8.1)$ & $21(6.8)$ & $8(16.3)$ & \\
\hline Histology (n, \%) & & & & 0.067 \\
\hline AIS & $35(9.7)$ & $29(9.3)$ & $6(12.2)$ & \\
\hline MIA & $50(13.9)$ & 40 (12.9) & $10(20.4)$ & \\
\hline W/M DA & $149(41.4)$ & $129(41.5)$ & $20(40.8)$ & \\
\hline PDA & $14(3.9)$ & $14(4.5)$ & $0(0.0)$ & \\
\hline Squamous carcinoma & $5(1.4)$ & $5(1.6)$ & $0(0.0)$ & \\
\hline Small cell carcinoma & $1(0.3)$ & $1(0.3)$ & $0(0.0)$ & \\
\hline Right middle lobe & $28(7.8)$ & $28(9.0)$ & $0(0.0)$ & \\
\hline Right lower lobe & $63(17.5)$ & $56(18.0)$ & $7(14.3)$ & \\
\hline Left upper lobe & $83(23.1)$ & $70(22.5)$ & $13(26.5)$ & \\
\hline Left lower lobe & $51(14.2)$ & $43(13.8)$ & $8(16.3)$ & \\
\hline Nodule type (n, \%) & & & & 0.109 \\
\hline Solid & $163(45.3)$ & $146(46.9)$ & $17(34.7)$ & \\
\hline Sub-solid & $197(54.7)$ & $165(53.1)$ & $32(65.3)$ & \\
\hline Spicule (n, \%) & & & & 0.477 \\
\hline No & $226(62.8)$ & $193(62.1)$ & $33(67.3)$ & \\
\hline Yes & $134(37.2)$ & $118(37.9)$ & $16(32.7)$ & \\
\hline
\end{tabular}

Table 1 (continued) 
Table 1 (continued)

\begin{tabular}{lccc}
\hline Characteristics & Total & Non-MDT & MDT \\
\hline Pathology (n, \%) & & & 0.304 \\
Malignant & $257(71.4)$ & $219(70.4)$ & $38(77.6)$ \\
Benign & $103(28.6)$ & $92(29.6)$ & $11(22.4)$ \\
SUV, median (min-max) & $1.0(0.5-4.4)$ & $1.6(0.5-3.3)$ & $1.0(1.0-4.4)$ \\
SUV sub-group (n, \%) & & & 0.843 \\
No PET-CT & $278(77.2)$ & $242(77.8)$ & $36(73.5)$ \\
$0-2.5$ & $79(22.0)$ & $67(21.5)$ & $12(24.5)$ \\
$\geq 2.5$ & $3(0.8)$ & $2(0.6)$ & $1(2.0)$ \\
\hline
\end{tabular}

MDT, multidisciplinary team; AIS, adenocarcinoma in situ; MIA, minimal invasive adenocarcinoma; PDA, poorly differentiated adenocarcinoma; W/M DA, well/moderately differentiated adenocarcinoma.

\section{Discussion}

\section{The conventional diagnosis and procedure of small lung nodules and their limitation}

The ability to distinguish malignant nodules from a benign nodule is becoming a research hotspot. Routinely, a radiologist will give a general diagnosis first based on the CT images. Next, if the nodule's malignancy is hard to predict, and if it is large enough, a PET-CT is recommended. If the SUV of the nodule is above 2.5 , the probability of malignancy is high. Thirdly, a patient needs a biopsy of the nodule, which is conducted by pulmonologists or medical oncologists; a pathological diagnosis is the gold standard. Lastly, if a malignancy of the nodules is confirmed, and the patient has an adequate cardiopulmonary function and is willing to undergo an operation, resection of the nodule will be conducted by a thoracic surgeon. From the conventional diagnosis procedure of lung nodules, we can conclude that radiologists, pulmonologists, or medical oncologists and surgeons acting in order before surgery; CT, PET-CT, biopsy and surgery play important roles in the differentiation of small pulmonary nodules. For those pulmonary nodules for which a biopsy is easy to perform, the conventional procedure works well. However, for those nodules in which acquiring a biopsy sample is difficult, such as those that are too small or located in a place where biopsy is inappropriate, the conventional techniques and procedures have limitations. PET-CT scans will show false-positives in some benign lesions, as seen in infections such as pneumonia and tuberculosis (14), which is more prevalent in China than in western countries. On the other hand, scans will show false-negative in tumors with low ${ }^{18} \mathrm{~F}$-FDG uptake, such as in lung adenocarcinoma in site and carcinoid tumors (14). In addition, with the decrease of the diameter of the nodules, the reliability of SUV decreases as well. PET-CT has little to no value in small lung nodules which are $\leq 1 \mathrm{~cm}$ (15), which is generally accepted as the smallest size that be accurately evaluated by PET-CT (14).

As to bronchoscopy and CT-guided transthoracic fine/ core-needle biopsy, which are well-established techniques for diagnosis, the failure of bronchoscopy is relatively high in lesions measuring $20 \mathrm{~mm}$ or smaller or if the lesion is in the outer third of the lung (16). Also, transthoracic percutaneous fine/core-needle aspiration biopsy has a considerable risk of complications, such as pneumothorax and bleeding (17). Tumor cell implantation along the needle tract is rare but it is a potentially extreme and serious complication (18). In addition, the two kinds of biopsy can only obtain part of the specimen of the nodule, which cannot reflect the whole nodule. Even if the pathological diagnosis of biopsy is benign, it does not mean the whole nodule is benign $(19,20)$. Even worse, they cannot always acquire adequate specimens from small lung nodules for gene mutation tests, which are the basis for target therapy for lung cancer nowadays. Even if the nodules are diagnosed as malignant, they still need to be removed by operation. In the era of minimally invasive surgery, surgeons tend to use an efficient one-step way to manage pulmonary nodules (21). Some progress has been made to improve the current situation. Video-assisted thoracoscopic surgery (VATS) has been developing quickly. The accuracy, low morbidity, and easy availability of the technique make it 
Table 2 Clinical characteristics of the 2 cohorts of patients with solitary pulmonary nodules identified for propensity score matching (based on pathology) before matching

\begin{tabular}{|c|c|c|c|c|}
\hline Characteristics & Malignant & Benign & PPV (\%) & $P$ value \\
\hline Age (years) & $55.0(48.0-64.0)$ & $52.0(45.0-56.5)$ & & $<0.001$ \\
\hline Nodule size (mm) & $13.0(10.0-17.0)$ & $12.0(9.0-15.0)$ & & 0.031 \\
\hline Gender (n, \%) & & & & $<0.001$ \\
\hline Female & $160(62.3)$ & $40(38.8)$ & 80.0 & \\
\hline History of smoking (n, \%) & & & & 0.720 \\
\hline No & $204(79.4)$ & $80(77.7)$ & 71.8 & \\
\hline Yes & $53(20.6)$ & $23(22.3)$ & 69.7 & \\
\hline Yes & $24(9.3)$ & $5(4.9)$ & 82.8 & \\
\hline Nodule position (n, \%) & & & & 0.145 \\
\hline Right upper lobe & $103(40.1)$ & $32(31.1)$ & 76.3 & \\
\hline Right middle lobe & $15(5.8)$ & $13(12.6)$ & 53.6 & \\
\hline Right lower lobe & $42(16.3)$ & $21(20.4)$ & 66.7 & \\
\hline Left upper lobe & $61(23.7)$ & $22(21.4)$ & 73.5 & \\
\hline Left lower lobe & $36(14.0)$ & $15(14.6)$ & 70.6 & \\
\hline Nodule type (n, \%) & & & & $<0.001$ \\
\hline MDT (n, \%) & & & & 0.304 \\
\hline NonMDT & $219(85.2)$ & $92(89.3)$ & 70.4 & \\
\hline MDT & $38(14.8)$ & $11(10.7)$ & 77.6 & \\
\hline SUV median (min-max) & $1.5(0.5-4.4)$ & $1.0(1.0-3.3)$ & & 0.993 \\
\hline SUV sub-group* (n, \%) & & & & 0.166 \\
\hline $0-2.5$ & $56(98.2)$ & $23(92.0)$ & & \\
\hline$\geq 2.5$ & $1(1.8)$ & $2(8.0)$ & & \\
\hline
\end{tabular}

*, in the total 360 patients, 278 patients didn't undergo PET-CT, so they don't have SUV value, the other 82 patients have SUV value. PPV, positive predictive value; MDT, multidisciplinary team.

very popular. Also, it can not only acquire a diagnosis, but it can also resect the nodules completely, supplying entire nodules for pathological tests and gene mutation tests $(22,23)$. Due to the reasons stated above, VATS is the most 
Table 3 Clinical characteristics of the two cohorts of patients with solitary pulmonary nodules identified after propensity score matching

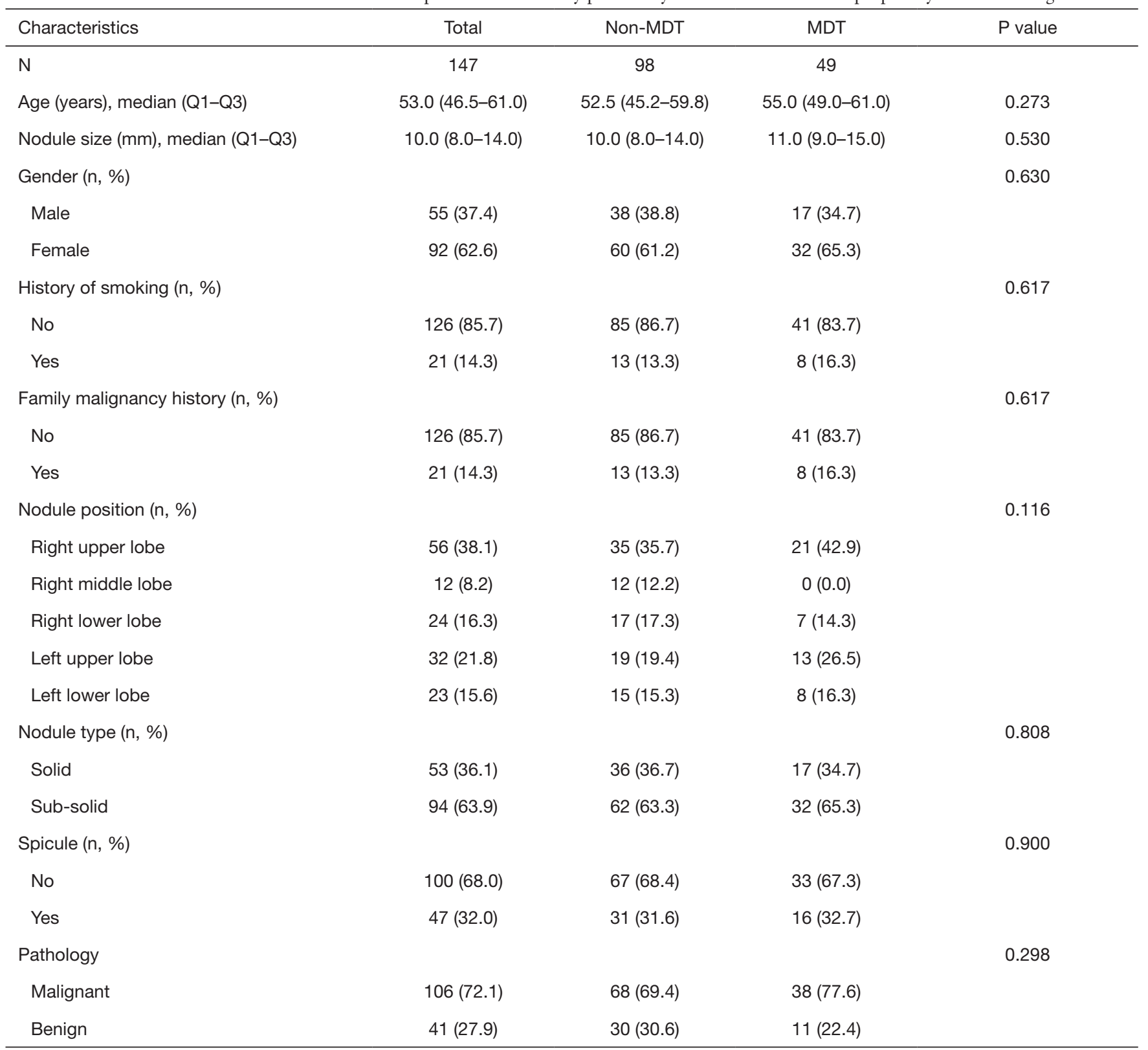

MDT, multidisciplinary team.

used procedure for small lung nodules in our hospital. Moreover, there is a study that shows that direct resection of the suspicious lung nodules without preoperative biopsy will not increase the incidence of resected benign pathology and may decrease surgical wait time (24). In this respect, it is important to predict the malignancy of the nodule without preoperative biopsy.

Whether the specialists can give the correct diagnosis depends on their knowledge and clinical experience. There are significant differences in the management of small pulmonary nodules among radiologists, pulmonologists, and thoracic surgeons. Radiologists tend to recommend short-term follow-up, while the pulmonologists and surgeons tend to recommend operation (25). Once the same patient receives two different recommendations, the proper diagnosis for the patient can be confusing. 
Table 4 Clinical characteristics of the 2 cohorts of patients with solitary pulmonary nodules identified for propensity score matching (based on pathology) after matching

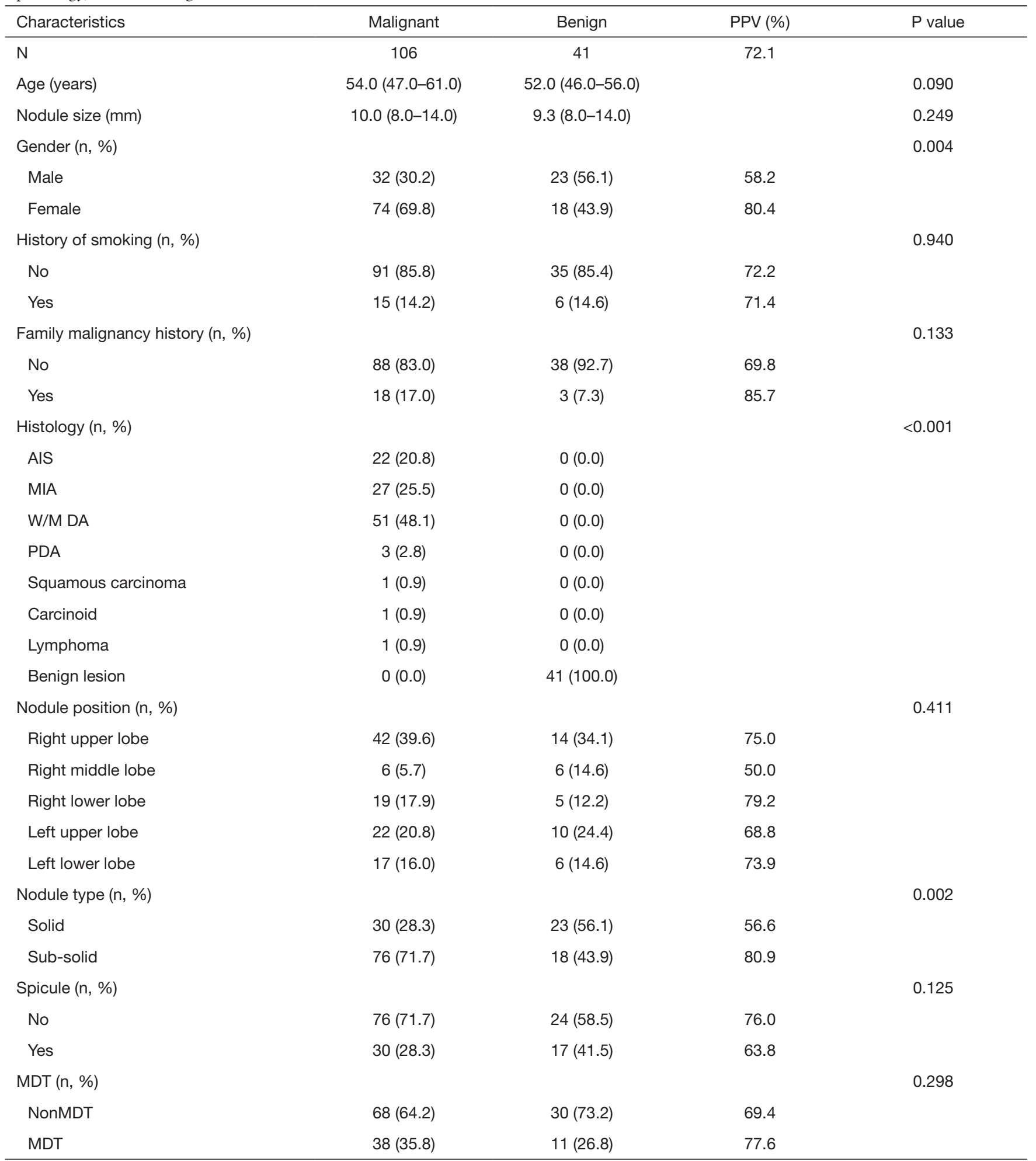

PPV, positive predictive value; MDT, multidisciplinary team; AIS, adenocarcinoma in situ; MIA, minimal invasive adenocarcinoma; PDA, poorly differentiated adenocarcinoma; W/M DA, well/moderately differentiated adenocarcinoma. 
Table 5 Clinical characteristics of participants (based on SUV)

\begin{tabular}{|c|c|c|c|}
\hline Characteristics & $0-2.5$ & $\geq 2.5$ & $P$ value \\
\hline $\mathrm{N}$ & 79 & 3 & \\
\hline Age (years) & 56.6 (10.1), $56.0(50.0-64.5)$ & 50.0 (7.9), $53.0(47.0-54.5)$ & 0.269 \\
\hline Male & $29(36.7)$ & $3(100.0)$ & \\
\hline Female & 50 (63.3) & $0(0.0)$ & \\
\hline No & $64(81.0)$ & $2(66.7)$ & \\
\hline Yes & $15(19.0)$ & $1(33.3)$ & \\
\hline Family malignancy history (n, \%) & & & 0.562 \\
\hline No & 71 (89.9) & $3(100.0)$ & \\
\hline MIA & $3(3.8)$ & $0(0.0)$ & \\
\hline W/M DA & $42(53.2)$ & $1(33.3)$ & \\
\hline PDA & $3(3.8)$ & $0(0.0)$ & \\
\hline Squamous carcinoma & $1(1.3)$ & $0(0.0)$ & \\
\hline Carcinoid & $2(2.5)$ & $0(0.0)$ & \\
\hline Benign lesion & $23(29.1)$ & $2(66.7)$ & \\
\hline Nodule position (n, \%) & & & 0.175 \\
\hline Right upper lobe & $22(27.8)$ & $2(66.7)$ & \\
\hline Right middle lobe & $5(6.3)$ & $1(33.3)$ & \\
\hline Sub-solid & $34(43.0)$ & $0(0.0)$ & \\
\hline Spicule (n, \%) & & & 0.418 \\
\hline No & $45(57.0)$ & $1(33.3)$ & \\
\hline Yes & $34(43.0)$ & $2(66.7)$ & \\
\hline Pathology (n, \%) & & & 0.166 \\
\hline Malignant & $56(70.9)$ & $1(33.3)$ & \\
\hline Benign & $23(29.1)$ & $2(66.7)$ & \\
\hline MDT (n, \%) & & & 0.398 \\
\hline NonMDT & $67(84.8)$ & $2(66.7)$ & \\
\hline MDT & $12(15.2)$ & $1(33.3)$ & \\
\hline Nodule size $(\mathrm{mm})(\mathrm{n}, \%)$ & & & 0.300 \\
\hline$<10$ & $21(26.6)$ & $0(0.0)$ & \\
\hline$\geq 10$ & $58(73.4)$ & $3(100.0)$ & \\
\hline
\end{tabular}

MDT, multidisciplinary team; AIS, adenocarcinoma in situ; MIA, minimal invasive adenocarcinoma; PDA, poorly differentiated adenocarcinoma; W/M DA, well/moderately differentiated adenocarcinoma. 
Table 6 Comparison of PPV between non-MDT and MDT groups after matching

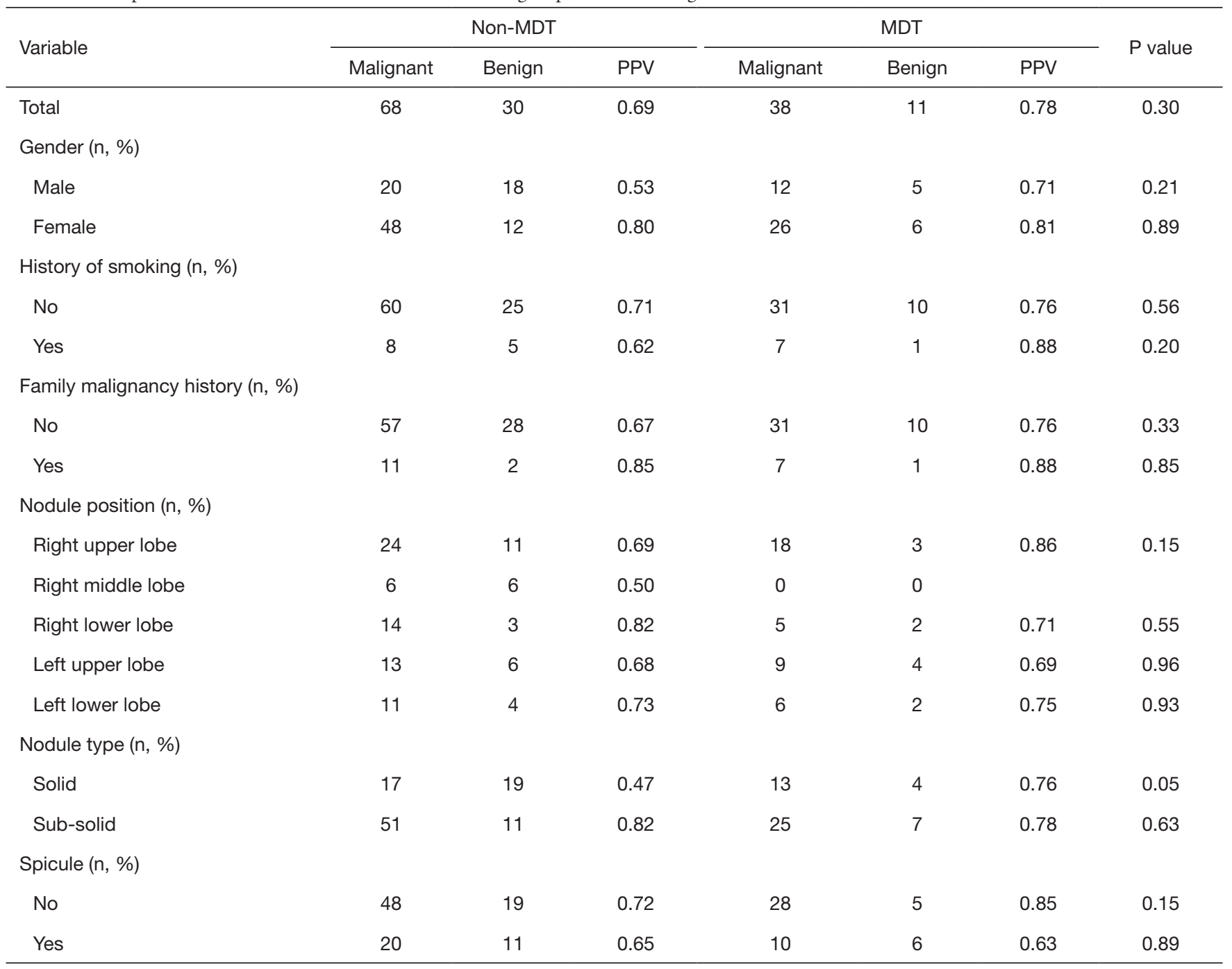

PPV, positive predictive value; MDT, multidisciplinary team.

\section{The function of MDT}

An MDT is becoming a standard recommendation for lung cancer and pulmonary nodules in international guidelines (10). In China, with the number of discoveries of small lung nodules increasing, hospitals at different levels have been more frequently establishing many MDTs for lung nodules recently. However, the evidence to support MDT is scarce and the existing research about MDT in lung cancer has focused on late-stage lung cancer and the outcome. Evidence from breast cancer, lung cancer, head and neck cancer, and rectal cancer has demonstrated that MDTs can function better at the management of cancer, by decreasing the time from diagnosis to treatment, improving patient satisfaction, reducing costs, and increasing the accuracy of staging rather than improving the outcome of cancer patients (26). As to small lung nodules, MDTs focus on the prediction of small lung nodules which entails extensive energy and time expenditure for specialists and patients alike. Are these costs worth it? As of now there is no standard answer for this question.

\section{Our study}

Most nodules whose diameters are bigger than $2 \mathrm{~cm}$ are malignant (27), and the value of SUV on PET-CT is more reliable than that for nodules whose diameter is less than 


\section{Page 12 of 15}

Table 7 Characteristics and results of follow-up

\begin{tabular}{|c|c|}
\hline Characteristics & Outcome \\
\hline $\mathrm{N}$ & 21 \\
\hline Age & $56.0(53.0-62.0)$ \\
\hline Nodule size (mm) & $10.0(9.0-16.0)$ \\
\hline Follow-up (month) & $12.0(10.0-19.0)$ \\
\hline PET-CT value & $0.0(0.0-2.7)$ \\
\hline \multicolumn{2}{|l|}{$\operatorname{Sex}(n, \%)$} \\
\hline Male & $13(61.9)$ \\
\hline Female & $8(38.1)$ \\
\hline \multicolumn{2}{|l|}{ Family malignancy history (n, \%) } \\
\hline No & $17(81.0)$ \\
\hline Yes & $4(19.0)$ \\
\hline \multicolumn{2}{|l|}{ History of smoking (n, \%) } \\
\hline No & $11(52.4)$ \\
\hline Yes & $10(47.6)$ \\
\hline \multicolumn{2}{|l|}{ Nodule position (n, \%) } \\
\hline Right upper lobe & $9(42.9)$ \\
\hline Right middle lobe & $3(14.3)$ \\
\hline Right lower lobe & $4(19.0)$ \\
\hline Left upper lobe & $3(14.3)$ \\
\hline Left lower lobe & $2(9.5)$ \\
\hline \multicolumn{2}{|l|}{ Nodule type (n, \%) } \\
\hline Solid & $5(23.8)$ \\
\hline Sub-solid & $16(76.2)$ \\
\hline \multicolumn{2}{|l|}{ Spicule (n, \%) } \\
\hline No & $18(85.7)$ \\
\hline Yes & $3(14.3)$ \\
\hline \multicolumn{2}{|l|}{ The results of the follow-up (n, \%) } \\
\hline Malignant & $3(14.3)$ \\
\hline No change/shrink/disappear & $16(76.2)$ \\
\hline Loss of follow-up & $2(9.5)$ \\
\hline
\end{tabular}

$2 \mathrm{~cm}$. Also, it is relatively easier to obtain biopsy from these nodules, and thus it is easier to form pathological diagnosis, making an appropriate treatment recommendation more likely. Only those whose nodules are relatively difficult to acquire biopsy from would be referred to attend our
Liu et al. MDT's role in the prediction of small solitary lung nodules

MDT. Therefore, in this study, we focused on the small pulmonary nodules whose diameter was $\leq 2 \mathrm{~cm}$ and for which a pathological diagnosis before surgery was difficult to acquire.

For the group of patients who attend the non-MDT representative traditional sequential diagnostic procedure of small lung nodules, when a small pulmonary nodule on CT image is found in the patient, he or she will go to visit a thoracic surgeon directly or visit pulmonologists and medical oncologists first. If the nodule is difficult to biopsy, or if the pulmonologists and medical oncologists think the nodule is highly suspect for malignancy and it is operable, they will refer the patient to visit a thoracic surgeon. The surgeon will then recommend them to follow-up or recommend surgical resection. The group of patients who attend MDT are recommended to MDT and their cases are discussed in the MDT meeting.

Propensity-score-matching was adopted during the process of analysis to obtain a more reliable conclusion. Confounding factors that were reported to influence the prediction of the small nodules were used to score each patient. These include age, gender, nodule position, nodule type, nodule size, spicule sign, and history of smoking. Before matching, family malignant history, and nodule size were unbalanced between the non-MDT group and MDT group $(\mathrm{P}<0.05)$ (Table 1). After matching, all the variables were distributed and balanced $(\mathrm{P}>0.05)$ (Table 3).

In our study, most of the small solitary malignant pulmonary nodules $(\leq 2 \mathrm{~cm})$ were adenocarcinoma and located on the right upper lobe, which is consistent with the previous report (28). More females had small pulmonary nodules before and after matching, which is also a commonly reported phenomenon (7).

There was no difference in SUV value between the malignant and the benign group; in other words, in small pulmonary nodules, differentiating malignancy by SUV value is not reliable, which is corroborated in many other studies $(14,15)$.

We noticed that about $30 \%$ of patients in both group had received surgery for benign lesions. In the earlier studies, this number varied from $6-34 \%(29,30)$. The number in our hospital is within this range and is relatively high. There are two reasons to explain this pattern. First, most patients did not receive a preoperative biopsy. Second, due to the psychological factors in our country, many patients with benign lesions request surgery because of the threat of cancer development. They are unwilling to follow-up, and they usually think it is better to immediately remove 
the lesion completely, or they will worry about it during the follow-up-a phenomenon which is mentioned in other studies $(25,29)$.

In some sense, the nodules in the MDT group were even harder to differentiate than those of the non-MDT group because only when at least two physicians have different opinions about the nodule's malignance would they refer the patient to attend our MDT. Even under these conditions, we can see from our study that the positive predictive value of the MDT group was higher than that of the non-MDT group whether before or after propensity matching or whether considered as a total or as separate demographic groups. After matching, the total disparity increased, from $7.2 \%$ to $8.2 \%$, which would result in the less unnecessary operation of the small pulmonary nodules; however, the difference was not significant $(\mathrm{P}=0.3)$. From a statistical point of view, this could be due to the small number of participants. If we increase the number of participants, the difference would perhaps acquire significance.

Normally, high PPV may lead to low NPV. However, in our study, from the results of the follow-up of the patients in MDT, the NPV was $76.2 \%$, which was as high as PPV (77.6\%). The NPV was even much higher than the reported $51 \%$ NPV of CT-guided transthoracic core-needle biopsy (TTNB) of another study (31). In that report, most of the diameters of nodules were larger than $2 \mathrm{~cm}$. After a median follow-up time [12.0 months (10.0-19.0 months)], although the number of follow-up patients was relatively small (21), the data was still quite impressive.

In short, for small pulmonary nodules, our MDT not only tends to give a higher PPV but also simultaneously maintains a high NPV. We believe the reasons for this are as follows. First, MDT pays much more attention to the patients. We have a full-time secretary who can collect a detailed history, and test or examine reports carefully. Because every specialist has to voice his or her opinion at the meeting, the specialist will pay much more attention to the patients discussed than in the outpatient department. After they receive the information of the patient in the MDT-WeChat group, they can search the necessary information on the internet, or they can carefully review the radiological images in the radiological system of our hospital in advance.

In contrast, due to the busy schedule in our out-patient department, our specialist spends much less time deciding by themselves. Secondly, the decision of operation for nonMDT patients is usually made by the thoracic surgeon based on findings on radiological pictures, while in MDT, the decision is made by all the specialists attending the MDT. Most radiologists usually only judge based on the characteristics of nodules including diameter, volume, margin, attenuation and location (32), while the oncologist who performs the CT-guided fine-needle aspiration or the respiratory specialist who performs the bronchoscopy have much more clinical experience to consider the pathology of the radiological image. Benign lesions, appear as small lung nodules and also have a malignant appearance on CT images, such as granulomatous inflammation, with a high SUV on PET-CT, include tuberculosis and pulmonary fungal infection occupying a large portion (33-35). In this case, respiratory specialists have more experience in verifying and treating them.

Other than diagnosis, the patients with small pulmonary nodules can also receive treatment benefits. As reported, in lung nodules, SABR can achieve a similar 5-year survival compared to an operation (36). Female non-smoking patients were the predominant population with pulmonary nodules. This Asian group has high probability of EGFR mutation (37), and thus oral EGFR-TKI has demonstrated great efficiency and convenience in their treatment. This group can receive one of the three following treatments: operation, SABR, or EGFR-TKI. Based on the probability of malignancy, the difficulty of operation, the patients' cardiopulmonary function, and the willingness to receive operation, radiologists, surgeons, radiation oncologists, and medical oncologists would weigh the pros and cons together and make a tailored recommendation for them. It would be unimaginable to see non-MDT patients receive as tailored a recommendation in such a short amount of time.

Along with the advantages above, MDT also offers other benefits. First, the specialists will learn from each other and together enhance their knowledge of pulmonary nodules. Secondly, the patients of MDT will feel more convinced by a conclusion of an MDT and feel more comfortable through the whole procedure because they have received adequate attention in the form of detailed explanation by the secretary and senior oncologist in charge and consolation and procedure instruction from the nurse. Overall, the patient receives better wholeprocess management which saves much time and energy. The sequential procedures are arranged and coordinated by experts during and after the meeting in a timely manner. For example, after the operation, based on perioperative and pathological findings, an oncologist will decide whether they should receive radiotherapy or chemotherapy without significant delay. Overall, being attended to by our MDT provides superior care for our patients.. 


\section{Page 14 of 15}

\section{Limitations}

Our study is the first of its kind to examine the diagnostic value of an MDT on small solitary pulmonary nodules $(\leq 2 \mathrm{~cm})$. The study used a unique propensity-score matching technique to provide first-time evidence on a novel subject in China. However, our research still has some limitations. Firstly, it is retrospective without significant long-term follow-up. We have not yet compared the survival outcome between the non-MDT group and the MDT group yet. Secondly, although our department is a top 20 cancer institute in China, this study only considered the experience of a single institute. Lastly, the sample size is small due to a short study period. Despite these limitations, we were still able to successfully demonstrate the powerful predictive accuracy of the MDT in small pulmonary nodules $(\leq 2 \mathrm{~cm})$.

\section{Conclusions}

Small solitary pulmonary nodules tend to be more correctly diagnosed with an MDT, especially the negative nodules. Therefore, an MDT can reduce the unnecessary resection of small pulmonary nodules, and it is worthwhile to promote this method in the hospitals of developing countries.

\section{Acknowledgments}

We thank all the cooperation of our MDT members and the patients. We thank Daniel Li for proofreading our manuscript.

\section{Footnote}

Conflicts of Interest: The authors have no conflicts of interest to declare.

Ethical Statement: The authors are accountable for all aspects of the work in ensuring that questions related to the accuracy or integrity of any part of the work are appropriately investigated and resolved. The study protocol was approved by the medical ethics committee of the Second Xiangya Hospital.

\section{References}

1. Siegel RL, Miller KD, Jemal A. Cancer statistics, 2018. CA Cancer J Clin 2018;68:7-30.
2. Allemani C, Matsuda T, Di Carlo V, et al. Global surveillance of trends in cancer survival 2000-14 (CONCORD-3): analysis of individual records for 37513025 patients diagnosed with one of 18 cancers from 322 population-based registries in 71 countries. Lancet 2018;391:1023-75.

3. Heineman DJ, Daniels JM, Schreurs WH. Clinical staging of NSCLC: current evidence and implications for adjuvant chemotherapy. Ther Adv Med Oncol 2017;9:599-609.

4. Murakami S, Ito H, Tsubokawa N, et al. Prognostic value of the new IASLC/ATS/ERS classification of clinical stage IA lung adenocarcinoma. Lung Cancer 2015;90:199-204.

5. Aberle DR, Adams AM, Berg CD, et al. Reduced lungcancer mortality with low-dose computed tomographic screening. N Engl J Med 2011;365:395-409.

6. Ost DE, Gould MK. Decision making in patients with pulmonary nodules. Am J Respir Crit Care Med 2012;185:363-72.

7. Gould MK, Tang T, Liu IL, et al. Recent Trends in the Identification of Incidental Pulmonary Nodules. Am J Respir Crit Care Med 2015;192:1208-14.

8. Zhou Z, Zhan P, Jin J, et al. The imaging of small pulmonary nodules. Transl Lung Cancer Res 2017;6:62-7.

9. Schreiber G, McCrory DC. Performance characteristics of different modalities for diagnosis of suspected lung cancer: summary of published evidence. Chest 2003;123:115S-128S.

10. MacMahon H, Naidich DP, Goo JM, et al. Guidelines for Management of Incidental Pulmonary Nodules Detected on CT Images: From the Fleischner Society 2017. Radiology 2017;284:228-43.

11. Callister ME, Baldwin DR, Akram AR, et al. British Thoracic Society guidelines for the investigation and management of pulmonary nodules. Thorax 2015;70 Suppl 2:ii1-ii54.

12. Floyd K, Glaziou P, Zumla A, et al. The global tuberculosis epidemic and progress in care, prevention, and research: an overview in year 3 of the End TB era. Lancet Respir Med 2018;6:299-314.

13. Liu C, Liu X, Wu F, et al. Using Artificial Intelligence (Watson for Oncology) for Treatment Recommendations Amongst Chinese Patients with Lung Cancer: Feasibility Study. J Med Internet Res 2018;20:e11087.

14. Ng YL, Patsios D, Roberts H, et al. CT-guided percutaneous fine-needle aspiration biopsy of pulmonary nodules measuring $10 \mathrm{~mm}$ or less. Clin Radiol 2008;63:272-7.

15. Khalaf M, Abdel-Nabi H, Baker J, et al. Relation between 
nodule size and 18F-FDG-PET SUV for malignant and benign pulmonary nodules. J Hematol Oncol 2008;1:13.

16. Baaklini WA, Reinoso MA, Gorin AB, et al. Diagnostic yield of fiberoptic bronchoscopy in evaluating solitary pulmonary nodules. Chest 2000;117:1049-54.

17. Portela de Oliveira E, Souza CA, Inacio JR, et al. Imagingguided Percutaneous Biopsy of Nodules $\leq 1 \mathrm{~cm}$ : Study of Diagnostic Performance and Risk Factors Associated With Biopsy Failure. J Thorac Imaging 2019. [Epub ahead of print].

18. Tomiyama N, Yasuhara Y, Nakajima Y, et al. CTguided needle biopsy of lung lesions: a survey of severe complication based on 9783 biopsies in Japan. Eur J Radiol 2006;59:60-4.

19. Savage C, Walser EM, Schnadig V, et al. Transthoracic Image-guided Biopsy of Lung Nodules: When Is Benign Really Benign? J Vasc Interv Radiol 2004;15:161-4.

20. Lee SM, Park CM, Lee KH, et al. C-arm cone-beam CTguided percutaneous transthoracic needle biopsy of lung nodules: clinical experience in 1108 patients. Radiology 2014;271:291-300.

21. Kuo SW, Tseng YF, Dai KY, et al. Electromagnetic Navigation Bronchoscopy Localization Versus Percutaneous CT-Guided Localization for Lung Resection via Video-Assisted Thoracoscopic Surgery: A PropensityMatched Study. J Clin Med 2019. doi: 10.3390/ jcm8030379.

22. Donahoe LL, Nguyen ET, Chung TB, et al. CT-guided microcoil VATS resection of lung nodules: a single-centre experience and review of the literature. J Thorac Dis 2016;8:1986-94.

23. Burdine J, Joyce LD, Plunkett MB, et al. Feasibility and value of video-assisted thoracoscopic surgery wedge excision of small pulmonary nodules in patients with malignancy. Chest 2002;122:1467-70.

24. Kaaki S, Kidane B, Srinathan S, et al. Is tissue still the issue? Lobectomy for suspicious lung nodules without confirmation of malignancy. J Surg Oncol 2018;117:977-84.

25. Prosch H, Strasser G, Oschatz E, et al. Management of patients with small pulmonary nodules: a survey of radiologists, pulmonologists, and thoracic surgeons. AJR Am J Roentgenol 2006;187:143-8.

26. Birchall M, Bailey D, King P, et al. Effect of process standards on survival of patients with head and neck cancer in the south and west of England. Br J Cancer 2004;91:1477-81

27. Kanemoto K, Satoh H, Ishikawa H, et al. Diagnostic procedures for small pulmonary nodules detected by mass- screening. Anticancer Res 2008;28:3153-5.

28. Liu Y, Wang H, Li Q, et al. Radiologic Features of Small Pulmonary Nodules and Lung Cancer Risk in the National Lung Screening Trial: A Nested Case-Control Study. Radiology 2018;286:298-306.

29. Rzyman W, Jelitto-Gorska M, Dziedzic R, et al. Diagnostic work-up and surgery in participants of the Gdansk lung cancer screening programme: the incidence of surgery for non-malignant conditions. Interact Cardiovasc Thorac Surg 2013;17:969-73.

30. Yung RC, Zeng MY, Stoddard GJ, et al. Transcutaneous computed bioconductance measurement in lung cancer: a treatment enabling technology useful for adjunctive risk stratification in the evaluation of suspicious pulmonary lesions. J Thorac Oncol 2012;7:681-9.

31. Fontaine-Delaruelle C, Souquet PJ, Gamondes D, et al. Negative Predictive Value of Transthoracic Core-Needle Biopsy: A Multicenter Study. Chest 2015;148:472-80.

32. Huang P, Park S, Yan R, et al. Added Value of Computeraided CT Image Features for Early Lung Cancer Diagnosis with Small Pulmonary Nodules: A Matched Case-Control Study. Radiology 2018;286:286-95.

33. Hou X, Zhang H, Kou L, et al. Clinical features and diagnosis of chronic pulmonary aspergillosis in Chinese patients. Medicine (Baltimore) 2017;96:e8315.

34. Cardinale L, Ardissone F, Novello S, et al. The pulmonary nodule: clinical and radiological characteristics affecting a diagnosis of malignancy. Radiol Med 2009;114:871-89.

35. Hsu J, Jia L, Pucar D, et al. Diffuse Idiopathic Pulmonary Neuroendocrine Cell Hyperplasia and Granulomatous Inflammation Mimicking High-Grade Malignancy on FDG-PET/CT. Clin Nucl Med 2017;42:47-9.

36. Huang K, Palma DA, Committee IART. Follow-up of patients after stereotactic radiation for lung cancer: a primer for the nonradiation oncologist. J Thorac Oncol 2015;10:412-9.

37. Yotsukura M, Yasuda H, Shigenobu T, et al. Clinical and pathological characteristics of EGFR mutation in operable early-stage lung adenocarcinoma. Lung Cancer 2017;109:45-51.

Cite this article as: Liu C, Zhao L, Wu F, Feng Y, Jiang R, $\mathrm{Hu} \mathrm{C}$. The multidisciplinary team plays an important role in the prediction of small solitary pulmonary nodules: a propensityscore-matching study. Ann Transl Med 2019;7(23):740. doi: 10.21037/atm.2019.11.125 